\title{
Single shot diffraction of picosecond 8.7-keV x-ray pulses
}

\author{
F. H. O'Shea, ${ }^{1, *}$ O. Williams, ${ }^{1}$ G. Andonian, ${ }^{1}$ S. Barber,${ }^{1}$ Y. Sakai,,${ }^{1}$ J. B. Rosenzweig, ${ }^{1}$ \\ I. Pogorelsky, ${ }^{2}$ M. Fedurin, ${ }^{2}$ K. Kusche, ${ }^{2}$ and V. Yakimenko ${ }^{2}$ \\ ${ }^{1}$ Department of Physics and Astronomy, University of California, Los Angeles, California 90095, USA \\ ${ }^{2}$ Accelerator Test Facility, Brookhaven National Laboratory, Upton, New York 11973, USA
}

(Received 26 July 2011; published 17 February 2012)

\begin{abstract}
We demonstrate multiphoton, single shot diffraction images of $\mathrm{x}$ rays produced by inverse Compton scattering a high-power $\mathrm{CO}_{2}$ laser from a relativistic electron beam, creating a pulse of $8.7 \mathrm{keV}$ x rays. The tightly focused, relatively high peak brightness electron beam and high photon density from the $2 \mathrm{~J} \mathrm{CO}_{2}$ laser yielded $6 \times 10^{7} \mathrm{x}$-ray photons over the full opening angle in a single shot. Single shot $\mathrm{x}$-ray diffraction is performed by passing the $\mathrm{x}$ rays though a vertical slit and on to a flat silicon (111) crystal. $10^{2}$ diffracted photons were detected. The spectrum of the detected $\mathrm{x}$ rays is compared to simulation. The diffraction and detection of $10^{2} \mathrm{x}$ rays is a key step to a more efficient time resolved diagnostic in which the number of observed $x$ rays might reach $10^{4}$; enabling a unique, flexible $\mathrm{x}$-ray source as a sub-ps resolution diagnostic for studying the evolution of chemical reactions, lattice deformation and melting, and magnetism.
\end{abstract}

DOI: 10.1103/PhysRevSTAB.15.020702

PACS numbers: $41.50 .+\mathrm{h}, 41.75 . \mathrm{Ht}, 41.85 . \mathrm{Qg}$

\section{INTRODUCTION}

High-brightness x-ray light sources are an invaluable tool for modern science. However, in addition to all the useful properties of $x$ rays created by insertion devices in synchrotrons [1], some applications demand sub-ps x-ray pulses. Short pulse x rays may be created in synchrotrons using laser slicing of the electron beam [2]. However, along with ultrashort $(\sim 100$ fs level [3]) pulses, inverse Compton scattering (ICS) sources have other attractive attributes such as straightforward x-ray energy tuning and polarization control as well as large peak flux [4]. The ICS source also benefits from the scaling of the scattered radiation wavelength with that of the interaction wavelength requiring a lower energy electron to produce a given wavelength as compared to undulators [5]. Further, unique aspects of ICS probes-production of up to $\mathrm{MeV} \gamma$ rays [6] and sub-ps measurement of delay between pump and $\mathrm{x}$-ray probe pulses [7] — will extend the range of processes that can be studied to higher energy and shorter time scales. ICS sources [8], made compact by rf photoinjector [9] and chirped pulse amplification laser [10] technologies, provide relatively high peak brightness, narrow spectrum $\mathrm{x}$ rays with average brightness limited by accelerator and laser repetition rates. All of these features benefit research into microscopic processes that evolve at the ps time scale or shorter, including chemical reactions, structural changes such as deformation and melting, and magnetic damping

\footnotetext{
*foshea@physics.ucla.edu
}

Published by the American Physical Society under the terms of the Creative Commons Attribution 3.0 License. Further distribution of this work must maintain attribution to the author(s) and the published article's title, journal citation, and DOI. and demagnetization. Additionally, the possibility of highbrightness $\mathrm{MeV} \gamma$-ray production by ICS may permit beneficial applications in cargo inspection and remote sensing of nuclear materials [11]. It is in this context that we present the detection of $10^{2}$ diffracted $\mathrm{x}$ rays as evidence of suitability of the ICS source as a time resolved $\mathrm{x}$-ray diagnostic.

The pulse length of ICS $\mathrm{x}$ rays is determined by the interaction geometry, which is typically head-on $\left(180^{\circ}\right)$ or orthogonal $\left(90^{\circ}\right)[5,12]$. In the head-on case the x-ray pulse length is determined by the electron pulse duration, which can be made as short as sub-ps using techniques such as magnetic chicane compression [13] or velocity bunching [14]. To demonstrate multiphoton production and detection, we have produced single shot images of Bragg diffraction of the $\mathrm{x}$ rays created at the ICS source developed at the Brookhaven National Laboratory Accelerator Test Facility (BNL ATF) [3,4] using a flat silicon crystal as a diffraction element.

\section{SINGLE SHOT DIFFRACTION}

The experimental setup is illustrated in Fig. 1, while the parameters for the electron beam and the $\mathrm{CO}_{2}$ laser at the interaction point (IP) are given in Table I. The $\mathrm{x}$ rays produced at the IP exit the vacuum system through a $250 \mu \mathrm{m}$ beryllium window; their path is determined by the direction of motion of the electrons [6,12]. After the IP the electrons are steered to a beam dump with a vertical dipole. An adjustable slit is placed directly downstream of the Be window to select $\mathrm{x}$ rays with small divergence in one transverse dimension. The slit is composed of two $5 \mathrm{~mm}$ thick aluminum plates controlled by independent linear translation stages. The $\mathrm{x}$-ray imaging system 


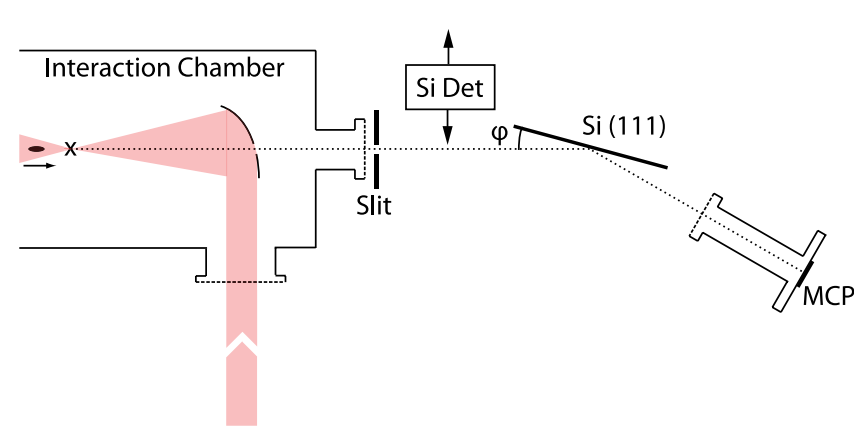

FIG. 1. Experimental setup of the ICS source at the BNL ATF. The $\mathrm{x}$ rays (dotted line) are generated at the IP $(\mathrm{X})$, collimated by the metal slits, and are then reflected off the silicon crystal, $\mathrm{Si}$ (111), for detection at the MCP. A retractable silicon detector (Si Det) is used for total flux measurements.

consists of a chevron-type microchannel plate (MCP) and a phosphor. The phosphor is imaged with a sensitive megapixel CCD fitted with a $50 \mathrm{~mm}$ focal length $(f / 1.3)$ lens. The $\mathrm{x}$ rays pass through a second $250 \mu \mathrm{m}$ Be vacuum window before illuminating the MCP.

A retractable, calibrated silicon diode is used to measure the total intensity of the x-ray flux [15]. The x rays passed through the vacuum window on the interaction chamber (see Fig. 1) and $20 \mathrm{~cm}$ of air before detection by the diode. The high average number of $\mathrm{x}$ rays created per shot, $6 \times 10^{7}$, was made possible by the interaction of a focused, intense electron beam with the large photon density of the $2 \mathrm{~J} \mathrm{CO}_{2}$ laser. We note that the $\mathrm{CO}_{2}$ laser has the advantage of lower photon energy when compared to solid state lasers, thus leading to larger x-ray counts for a given total laser energy (e.g. a 14-fold increase over $800 \mathrm{~nm}$ lasers) [4]. The measured total photon flux of $6 \times 10^{7}$ photons is in excellent agreement with the analytical estimate of $7 \times 10^{7}$ given by the approach of Ref. [6], that assumes the classical Thomson cross section and a single collision luminosity.

TABLE I. Electron beam and $\mathrm{CO}_{2}$ laser parameters at the interaction point.

\begin{tabular}{lc}
\hline \hline Parameter & Value \\
\hline Electron Beam & \\
Energy $(\mathrm{MeV})$ & 70.0 \\
Charge $(\mathrm{pC})$ & 200 \\
Energy spread rms & $0.1 \%$ \\
Bunch length rms (ps) & 4.0 \\
Normalized emittance rms (mm mrad) & 1 \\
Spot size rms $(\mu \mathrm{m})$ & 30 \\
CO ${ }_{2}$ Laser & \\
Energy $(\mathrm{J})$ & 2 \\
Wavelength $(\mu \mathrm{m})$ & 10.6 \\
Pulse length FWHM (ps) & 6.0 \\
Waist size FWHM $(\mu \mathrm{m})$ & 140 \\
Bandwidth FWHM & $0.7 \%$ \\
\hline \hline
\end{tabular}

This total flux corresponds to a peak brightness of $3.2 \times 10^{19}$ photons $/\left[\mathrm{s} \mathrm{mm}^{2} \mathrm{mrad}^{2}(10 \% \mathrm{BW})\right]$. ICS sources produce a large relative bandwidth due to the redshift of off-axis-directed photons. The total bandwidth can be narrowed using an aperture which will simultaneously sacrifice flux [3]. Simulation (following Ref. [12]) indicates that an aperture of $0.5 \mathrm{mrad}$ half angle will pass $2.7 \%$ of the photons having a $1.1 \%$ relative bandwidth for a peak brightness of $1.6 \times 10^{19}$ photons/ $\left[\mathrm{s} \mathrm{mm}^{2} \operatorname{mrad}^{2}(0.1 \% \mathrm{BW})\right]$. Because of the modest efficiency of our detection scheme, we elected not to use an aperture this narrow.

Downstream of the IP, the diffraction of the narrow band $x$ rays from a 4 inch diameter silicon (111) crystal (see Fig. 1), mounted on a rotation stage, obeys the Bragg criterion: $\varepsilon_{x}=\hbar \pi c /(d \sin \varphi)$ [16]. Here, $\hbar$ and $c$ are the reduced Planck constant and the speed of light, respectively, $d$ is the spacing between the crystal planes, and $\varphi$ is the complement to the angle of incidence of the $\mathrm{x}$ rays on the crystal (as defined in the Bragg formulation). The angular deviation from the Bragg criterion, or angular acceptance [17], is negligible for the $\mathrm{Si}(111)$ crystal within the energy range analyzed; we thus assume that all $\mathrm{x}$ rays meeting the Bragg criteria are reflected with equal intensity.

A silicon crystal was chosen because of the wide availability of high quality samples. The sample used is a $0.5 \mathrm{~mm}$ thick single side polished crystal with surface roughness less than $5 \AA$ on the polished side, as specified by the manufacturer. To keep bending to a minimum, the crystal is held in place by a set of grooved jaws which are closed only until movement is arrested. Bisection of the laser by the rotated crystal $\left(\varphi=0^{\circ}\right)$ showed no visible bending of the crystal. The crystal was aligned to the $\mathrm{x}$ rays via alignment laser which was also used to define the electron beam path, measured using yttrium aluminum garnet fluorescent screens.

As the bandwidth of the $\mathrm{x}$ rays is quite large compared to the crystal acceptance, the spectral selection of the diffraction process, along with the upstream slit, limits the number of detected photons. The regularly spaced Laue spots [16] usually present in standard diffraction techniques are not produced; instead, the photons produce a characteristic diffraction pattern which is a function of the single electron $\mathrm{x}$-ray intensity distribution and the electron beam distribution, filtered by the diffraction process.

An example single shot diffraction image taken at $\varphi=13.25^{\circ}(8.6 \mathrm{keV})$ is shown in Fig. 2. The pattern is not vertically symmetric because the crystal was tilted slightly downward vertically (relative to the electron propagation through the IP), a systematic effect due to differences in transport between the electron beam and the alignment laser and a slight deflection of the electron beam by the downstream vertical dipole. This misalignment can be described by a vertical rotation of the crystal 


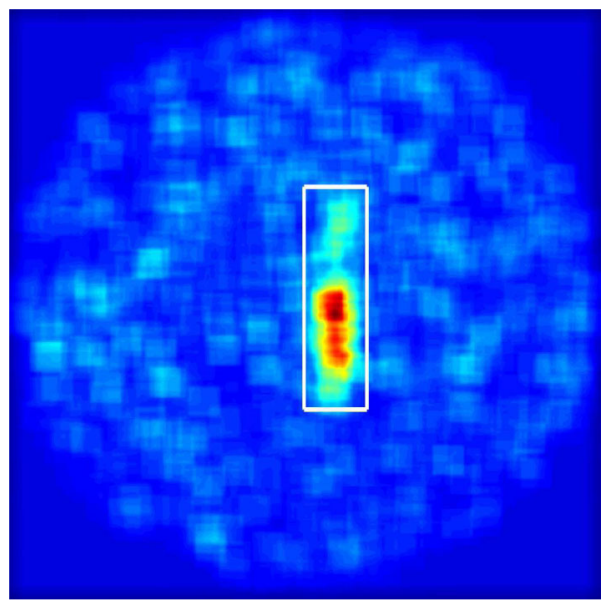

FIG. 2. A single shot diffraction image taken at $\varphi=13.25^{\circ}$ $(8.6 \mathrm{keV})$, median filtered. The white box indicates the diffraction pattern. The width (horizontal) of the x-ray distribution pattern is due to the slit acceptance while the vertical extent is determined by the Bragg criterion.

that precedes the rotation of the crystal to set the Bragg reflection angle. The diffraction element begins with planes normal to incident $\mathrm{x}$ rays, it is then rotated vertically by an angle $\eta$ (rotation down corresponds to positive $\eta$ ), after which it is rotated horizontally into a Bragg angle $\varphi$. The Bragg criteria for an incident $\mathrm{x}$ ray is

$$
\begin{aligned}
\frac{\lambda_{x}}{2 d}= & \cos (\eta) \cos (\varphi) \sin \left(\psi_{x}\right)+\sin (\eta) \sin \left(\psi_{y}\right) \\
& +\cos \eta \sin \varphi \sqrt{1-\sin ^{2}\left(\psi_{x}\right)-\sin ^{2}\left(\psi_{y}\right)},
\end{aligned}
$$

where $\lambda_{x}=h c / \varepsilon_{x}$ and $\psi_{(x, y)}$ are the horizontal and vertical angle of incidence on the crystal, respectively. The reflected $\mathrm{x}$ ray appears on the detecting screen with a vertical offset (relative to ideal alignment) of

$$
\vartheta-\vartheta_{0}=-\sin (2 \eta)\left(\varphi-\varphi_{0}\right)
$$

for small Bragg angles, measured from a relative zero, $\vartheta_{0}$, that occurs at $\varphi_{0}$. Vertical displacement of the expected null in the $\mathrm{x}$-ray distribution indicates that the vertical tilt of the crystal is approximately $40 \mathrm{mrad}$. This vertical tilt is consistent with the ratio of the intensity of the upper and lower peaks in the diffraction patterns.

The vertical extent of the slit allows the full angularspectral distribution of the photons to pass through, while the slit limits the horizontal extent to $\pm 0.5 \mathrm{mrad}$. The result is that the diffraction pattern displays a vertical distribution described by the parts of the photon beam that match the Bragg criteria for the angle of photon incidence. The largest photon flux after reflection occurs at $\varphi=13.25^{\circ}(8.6 \mathrm{keV})$. This angle setting produced the most intense diffraction patterns, indicating that the angle of incidence meets the Bragg criteria near the peak spectral intensity of the x-ray distribution. At this angle $83 \pm 22$

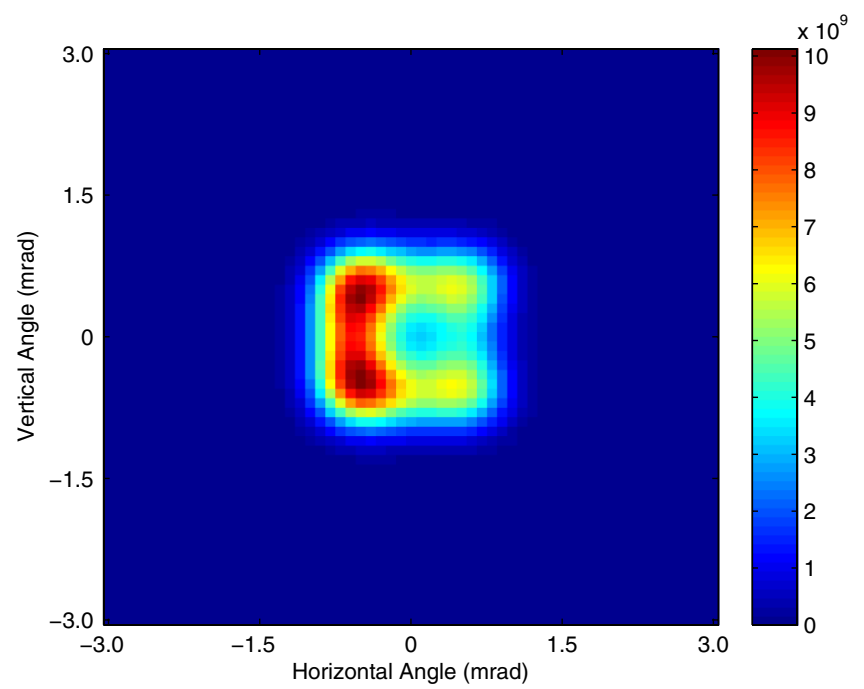

FIG. 3. Result of simulation of a single shot diffraction pattern for the same conditions as given in Fig. 2. The intensity is photons $/ \mathrm{rad}^{2}$.

photons are detected over the full aperture of the slit. A peak counting routine is used to identify counts on the MCP, with a threshold value to prevent counting of local variation in noise. It is assumed that each local maximum is a single photon. Background counts are measured by averaging the total counts in other areas of equal size on the detector, consistent with background measurements taken with the source turned off.

The error in the calculated number of $\mathrm{x}$ rays detected at the MCP is due to differences in laser energy from shot to shot. Including the attenuation of $70 \mathrm{~cm}$ of air, the transmission of the beryllium windows (96\% [18]) and the efficiency of the MCP ( $\approx 1 \%$ [3]), we estimate, using the given parameters, that the crystal reflects up to $10^{4}$ photons, while $10^{2}$ are detected. To confirm this estimate a numerical code based on the semianalytical calculation found in Ref. [6] was used with the x-ray energy related to the emission angle via Eq. (1); the result is that $2 \times 10^{4}$ photons are reflected. The photon density distribution can be seen in Fig. 3. The difference between the patterns seen in Figs. 2 and 3 is attributed to the deviation of the electron and laser beams from Gaussian distributions, which are assumed in the semianalytical treatment, and the presence of the slit. The detected flux is a dramatic improvement over the most noted ICS diffraction experiment which yielded only approximately a single detected photon per interaction [2].

\section{X-RAY SPECTRUM}

Characterization of the ICS x-ray source requires measurements of the electron and laser beam properties at the interaction point (IP), which can be used to supplement direct measurements of the $\mathrm{x}$-ray spectral distribution via simulation $[6,12]$. Prior ICS work has studied the electron 
beam phase space distribution [19], and measured the bandwidth of the $\mathrm{x}$ rays by using the transmission spectra of transition metals to preferentially attenuate the $\mathrm{x}$ rays [3]. We supplement the previous work by using the diffracted $\mathrm{x}$ rays to quantify the near-axis bandwidth of the ICS photons over a $1 \mathrm{keV}$ range of $\mathrm{x}$-ray energies centered around $8.7 \mathrm{keV}$ and compare the results to simulation [12].

To begin an enumeration of the sources of ICS x-ray bandwidth, we start with the angle-energy correlation of a single electron, single photon interaction, given by $[5,12]$ :

$$
\varepsilon_{x}=\frac{2 \gamma^{2} \varepsilon_{L}(1-\cos \phi)}{1+a_{L}^{2}+\gamma^{2}\left(\psi_{x}^{2}+\psi_{y}^{2}\right)}
$$

where $\varepsilon_{x}$ is the x-ray energy, $\varepsilon_{L}$ is the laser photon energy, $\gamma$ is the relativistic factor of the electron, and $a_{L}$ is the rootmean-square normalized vector potential of the laser at the interaction point. The observation angles of the $\mathrm{x}$ rays, as measured from the direction of propagation of the electron beam, in the horizontal $(x)$ and vertical $(y)$ directions are indicated by $\psi_{(x, y)}$, while $\phi$ is the angle of incidence of the laser with respect to the direction of forward propagation of the electrons. The source is configured for headon interaction $\left(\phi=180^{\circ}\right)$. We assume, consistent with measured laser parameters, that $a_{L}$ is very small and ignorable.

The diffraction criteria given by Bragg's law [Eq. (1)] and the angle-energy correlation given by Eq. (3), along with the acceptance limits given by the upstream slit, create a diffraction pattern which contains two lobes. The vertical misalignment of the silicon crystal suppresses the upper peaks on the low energy side in Fig. 4(a).

Determination of the spectral intensity of the $\mathrm{x}$ rays requires numerical simulations for realistic cases [6,12]. Following Ref. [12], we estimate the bandwidth due to the moments in the electron and laser beams: beam divergence at the IP (caused by the tight focus), beam energy spread, and laser bandwidth, respectively:

$$
\frac{\Delta \varepsilon_{x}}{\varepsilon_{x}} \approx \sqrt{4\left(\frac{\epsilon_{n}}{\sigma}\right)^{2}+4\left(\frac{\Delta \gamma}{\gamma}\right)^{2}+\left(\frac{\Delta \varepsilon_{L}}{\varepsilon_{L}}\right)^{2}}
$$

where $\epsilon_{n}$ is the normalized emittance and $\sigma$ is the transverse electron beam size, measured to be approximately symmetric. The laser focus, produced by an off-axis parabolic mirror $(f / 5)$, is a negligible source of $\mathrm{x}$-ray bandwidth relative to the above sources. Table II compares the sources of bandwidth in the detected $\mathrm{x}$ rays, as calculated using Eq. (4) and the ICS simulation found in Ref. [12]. The electron spot size is very small, $30 \mu \mathrm{m} \mathrm{rms}$, compared to the IP-to-detector distance, $2.15 \mathrm{~m}$, so the $\mathrm{x}$ rays are treated as originating from a point source. The bandwidth allowed by the opening angle of the detection scheme is
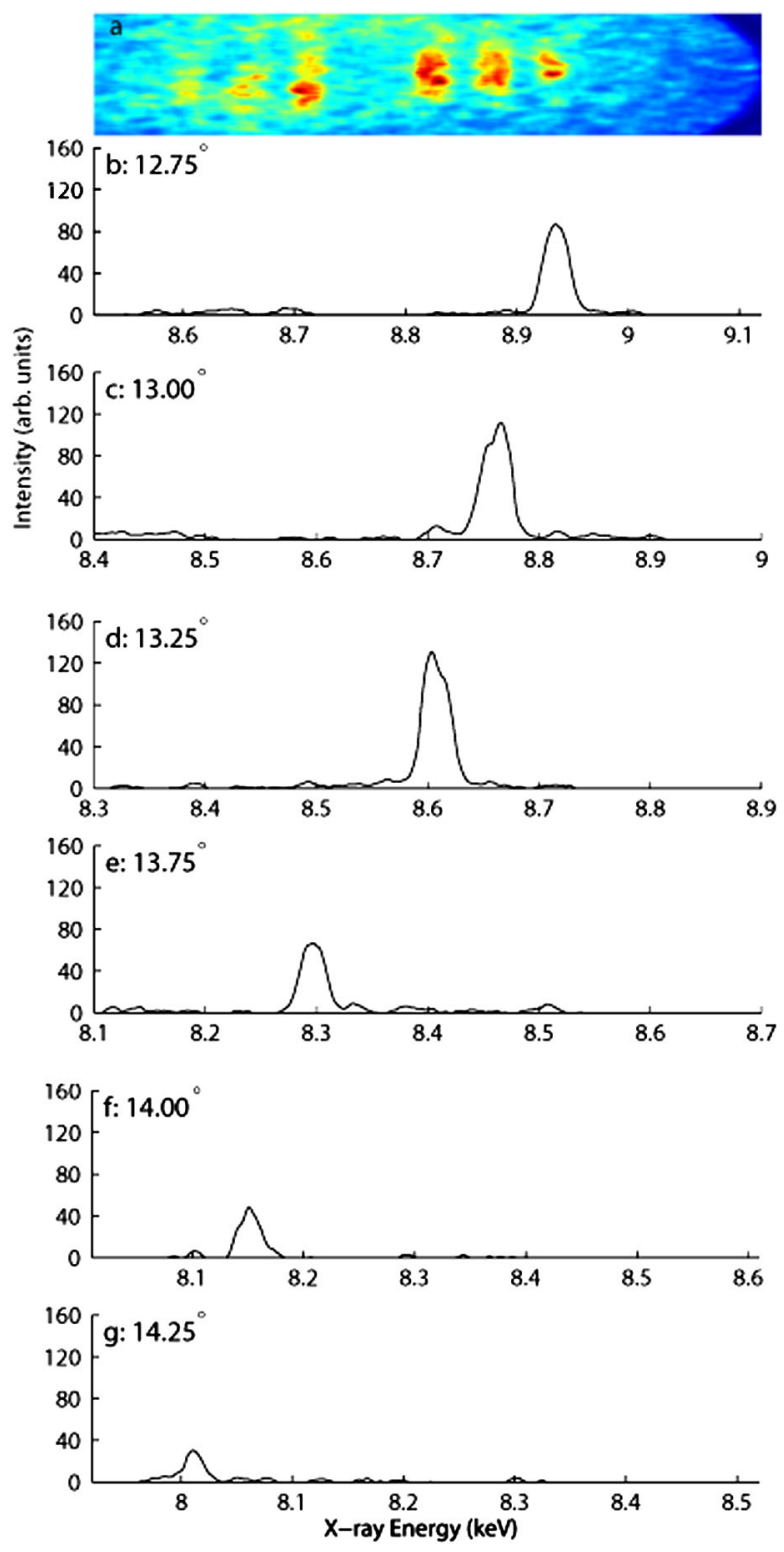

FIG. 4. (a) False color addition of 37 images taken at multiple angles and median filtered showing the physical relationship between the diffraction patterns at each angle $(\varphi)$ setting. The round edge of the MCP can be seen in the corners. The image is $10 \mathrm{mrad}$ tall. The vertical stripe pattern is due to the rotation of the silicon crystal (see Fig. 1). (b)-(g) Integrated lineouts from each angle $(\varphi)$ as noted. The integrated area is $2 \mathrm{mrad}$ vertically, centered on the pair of peaks seen in the $12.75^{\circ}$ diffraction pattern. Each angle lineout plot is the sum of 4-7 images which has been median filtered. The vertical scales of the images (b)-(g) are the same, while the range of abscissa has been changed to align the peaks to the distributions in (a).

estimated by assuming the radiation distribution is a symmetric Gaussian [1].

To measure the bandwidth of the ICS interaction, several images were produced at each of multiple crystal angle settings. The sampling of the spectrum within a window 
TABLE II. Comparison of contributions to $\mathrm{x}$-ray spectrum bandwidth between analytical estimates and simulation, both as found in [12].

\begin{tabular}{lcc}
\hline \hline Parameter & Estimate & Simulation \\
\hline Electron beam focusing & $0.4 \%$ & $0.3 \%$ \\
Energy spread & $0.2 \%$ & $0.2 \%$ \\
Laser bandwidth & $0.3 \%$ & $0.4 \%$ \\
Opening angle of detection & $2.5 \%^{\mathrm{a}}$ & $2.6 \%$ \\
\hline \hline
\end{tabular}

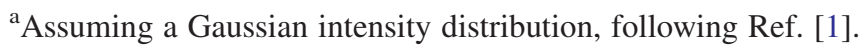

$2 \mathrm{mrad} \times 1 \mathrm{mrad}$ centered on the central axis of the electron beam is shown in Fig. 4(b).

Figure 5 shows a comparison between simulation and the measured data of the detected $\mathrm{x}$ rays; the blue line represents the output of simulation of the x-ray flux detected within the $2 \mathrm{mrad} \times 1 \mathrm{mrad}$ window using the parameters in Table I, while the circles are derived from median filtering each of the 37 images, integrating the total intensity over the $2 \mathrm{mrad} \times 1 \mathrm{mrad}$ aperture at each angle setting and taking the mean value; the error bars are the standard deviation of the mean of samples at each angle. The simulation curve is normalized for direct comparison to the measured data, which is normalized such that the $13.25^{\circ}$ data point lies on the simulation curve. The simulation curve, produced assuming ballistic motion of the electrons $\left(a_{L}=0\right)$, is not offset in abscissa, consistent with the previous assumption of negligible redshifting due to nonlinear electron motion. The simulation curve yields an rms bandwidth of $2.6 \%$ with a peak value of $8.72 \mathrm{keV}$. It is apparent that the bandwidth is largely due to the detection aperture which can be decreased at the expense of lowering the total photon count.

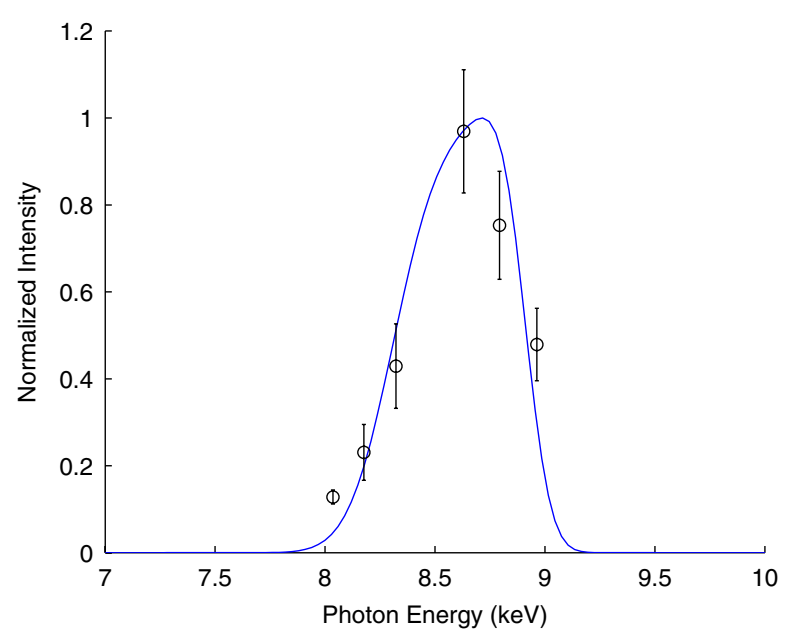

FIG. 5. Comparison of simulation (blue curve) and the integrated intensity of the recorded diffraction patterns (black circles). The error bars are the standard deviation of the mean normalized intensity at each angle.

\section{CONCLUSION}

In summary, we have produced single shot multiphoton diffraction images from an x-ray ICS source. The analytical estimates and predictions of simulation agree with the measured properties of the $\mathrm{x}$ rays. This demonstration of the ability to consistently produce and detect $10^{2}$ relatively high peak brightness $\mathrm{x}$ rays with ps-scale pulse length is an important step forward in the research of ultrafast processes (sub-ps) and nuclear material detection that is currently beyond the reach of modern third generation sources. Previously demonstrated electron bunch compression [3] as well as increased detector efficiency will allow the detection of nearly $10^{4}$ photons within a sub-ps pulse, capable of future, time dependent, diffraction studies. The compact size of the source will make tunable, ultrafast, hard $\mathrm{x}$ rays available on a university laboratory scale, so opening the door for a new class of pump-probe experiments in this context.

\section{ACKNOWLEDGMENTS}

The authors wish to thank P. Musumeci, J. Moody, E. Hemsing, and B. O'Shea for useful conversations. This work was supported by the Defense Threat Reduction Agency under Contract No. HDTRA1-10-0073, U.S. Department of Energy under Contracts No. DE-FG0207ER46272 and No. DE-FG03-92ER40693 and the Office of Naval Research under Contract ONR N0001406-1-0925.

[1] K. J. Kim, AIP Conf. Proc. 184, 565 (1989).

[2] R. W. Schoenlein et al., Science 274, 236 (1996); 287, 2237 (2000).

[3] O. B. Williams, Master's thesis, University of California, Los Angeles, 2009; O. Williams et al., Nucl. Instrum. Methods Phys. Res., Sect. A 608, S18 (2009).

[4] I. Pogorelsky et al., Phys. Rev. ST Accel. Beams 3, 090702 (2000).

[5] P. Sprangle, A. Ting, E. Esarey, and A. Fisher, J. Appl. Phys. 72, 5032 (1992).

[6] C. Sun and Y. K. Wu, Phys. Rev. ST Accel. Beams 14, 044701 (2011).

[7] C. M. Scoby, P. Musumeci, J. T. Moody, and M. S. Gutierrez, Phys. Rev. ST Accel. Beams 13, 022801 (2010).

[8] S. G. Anderson et al., Appl. Phys. B 78, 891 (2004).

[9] B. E. Carlsten, Nucl. Instrum. Methods Phys. Res., Sect. A 285, 313 (1989).

[10] D. Strickland and G. Mourou, Opt. Commun. 56, 219 (1985).

[11] W. Bertozzi et al., Phys. Rev. C 78, 041601(R) (2008).

[12] W. J. Brown and F. V. Hartemann, Phys. Rev. ST Accel. Beams 7, 060703 (2004).

[13] G. Andonian et al., Int. J. Mod. Phys. A 22, 4101 (2007). 
[14] M. Ferrario et al., Phys. Rev. Lett. 104, 054801 (2010)

[15] Detector details available at http://www.bnl.gov/atf/ systems/diagnostics/silicon_detector_for_xray.pdf.

[16] J.M. Cowley, Diffraction Physics (North-Holland, Amsterdam, 1975).
[17] J. Als-Nielsen and D. McMorrow, Elements of Modern $X$-Ray Physics (John Wiley, New York, 2000).

[18] Center for X-ray Optics: http://www.cxro.lbl.gov/.

[19] W.P. Leemans et al., Phys. Rev. Lett. 77, 4182 (1996). 